\title{
Confidence intervals for the common coefficient of variation of rainfall in Thailand
}

\author{
Warisa Thangjai ${ }^{1}$, Sa-Aat Niwitpong ${ }^{\text {Corresp., } 2}$, Suparat Niwitpong ${ }^{3}$ \\ ${ }^{1}$ Department of Statistics, Faculty of Science, Ramkhamhaeng University, Bangkok, Thailand \\ 2 Department of Applied Statistics, Faculty of Applied Science, King Mongkut's University of Technology North Bangok, Bangkok, Bangkok, Thailand \\ 3 Department of Applied Statistics, Faculty of Applied Science, King Mongkut's University of Technology North Bangok, Bangkok, Thailand \\ Corresponding Author: Sa-Aat Niwitpong \\ Email address: sa-aat.n@sci.kmutnb.ac.th
}

The log-normal distribution is often used to analyze environmental data like daily rainfall amounts. The rainfall is of interest in Thailand because high variable climates can lead to periodic water stress and scarcity. The mean, standard deviation or coefficient of variation of the rainfall in the area is usually estimated. The climate moisture index is the ratio of plant water demand to precipitation. The climate moisture index should use the coefficient of variation instead of the standard deviation for comparison between areas with widely different means. The larger coefficient of variation indicates greater dispersion, whereas the lower coefficient of variation indicates the lower risk. The common coefficient of variation, is the weighted coefficients of variation based on $\$ k \$$ areas, presents the average daily rainfall. Therefore, the common coefficient of variation is used to describe overall water problems of $\$ \mathrm{k} \$$ areas. In this paper, we propose four novel approaches for the confidence interval estimation of the common coefficient of variation of log-normal distributions based on the fiducial generalized confidence interval (FGCI), method of variance estimates recovery (MOVER), computational, and Bayesian approaches. A Monte Carlo simulation was used to evaluate the coverage probabilities and average lengths of the confidence intervals. In terms of coverage probability, the results show that the FGCI approach provided the best confidence interval estimates for most cases except for when the sample case was equal to six populations $(\$ k=\$ 6)$ and the sample sizes were small $\left(\$ n \_\{i\}<\$ 50\right)$, for which the MOVER confidence interval estimates were the best. The efficacies of the proposed approaches are illustrated with example using real-life daily rainfall datasets from regions of Thailand. 


\title{
Confidence Intervals for the Common Coefficient of Variation of Rainfall in Thailand
}

${ }_{4}$ Warisa Thangjai ${ }^{1}$, Sa-Aat Niwitpong ${ }^{2}$, and Suparat Niwitpong ${ }^{2}$

${ }_{5}{ }^{1}$ Department of Statistics, Faculty of Science, Ramkhamhaeng University, Bangkok, 10240, Thailand

${ }^{2}$ Department of Applied Statistics, Faculty of Applied Science, King Mongkut's

University of Technology North Bangkok, Bangkok, 10800, Thailand

Corresponding author:

Sa-Aat Niwitpong ${ }^{a}$

Email address: sa-aat.n@sci.kmutnb.ac.th

\begin{abstract}
The log-normal distribution is often used to analyze environmental data like daily rainfall amounts. The rainfall is of interest in Thailand because high variable climates can lead to periodic water stress and scarcity. The mean, standard deviation or coefficient of variation of the rainfall in the area is usually estimated. The climate moisture index is the ratio of plant water demand to precipitation. The climate moisture index should use the coefficient of variation instead of the standard deviation for comparison between areas with widely different means. The larger coefficient of variation indicates greater dispersion, whereas the lower coefficient of variation indicates the lower risk. The common coefficient of variation, is the weighted coefficients of variation based on $k$ areas, presents the average daily rainfall. Therefore, the common coefficient of variation is used to describe overall water problems of $k$ areas. In this paper, we propose four novel approaches for the confidence interval estimation of the common coefficient of variation of log-normal distributions based on the fiducial generalized confidence interval ( $F G C l)$, method of variance estimates recovery (MOVER), computational, and Bayesian approaches. A Monte Carlo simulation was used to evaluate the coverage probabilities and average lengths of the confidence intervals. In terms of coverage probability, the results show that the $\mathrm{FGCl}$ approach provided the best confidence interval estimates for most cases except for when the sample case was equal to six populations $(k=6)$ and the sample sizes were small $\left(n_{i}<50\right)$, for which the MOVER confidence interval estimates were the best. The efficacies of the proposed approaches are illustrated with example using real-life daily rainfall datasets from regions of Thailand.
\end{abstract}

\section{INTRODUCTION}

Droughts and floods are regular natural disasters in Thailand. Droughts occur when the hot season begins after a year with unusually little rainfall. Moreover, floods happen nearly every year during the monsoon season. The monsoon seasons in the country are distinct by region. Thailand is divided into six geographical regions such as the north, the northeast, the west, the central, the east, and the south. Various regions are prone to seasonal flash-flooding. The floods often occur in the north, the northern east, and the south. Since rainfall varies greatly depending on region and season. Therefore, the common coefficient of variation is used to represent the rainfall dispersion in different regions.

The log-normal distribution, widely used to describe the distribution of right-skewed data, has been used to model various real-life applications (Jafari and Abdollahnezhad, 2015). For instance, in climate sciences and hydrology, the rainfall measurements are right-skewed (Thangjai et al., 2020a). The coefficient of variation of the log-normal distribution depends on the variance $\left(\sigma^{2}\right)$ only (Thangjai et al, 2016) whereas the coefficient of variation of normal distribution depends on the mean $(\mu)$ and $\sigma^{2}$. Although the normal distribution is more well-known than the log-normal distribution in the natural and social sciences, the latter has been used in many applications. Examples of quantities which have 
approximate log-normal distributions include the particulate matter and rainfall frequency. Rumburg et al. (2001) studied the statistical distributions of daily particulate matter data from Spokane, Washington from January 1995 to December 1997. They found that the PM2.5 data is best fit by a three parameter log-normal distribution. Thangjai et al. (2020a) constructed the simultaneous confidence intervals for all differences of coefficients of variation of daily rainfall data on 17 July 2018 in different regions of Thailand. The daily rainfall data is log-normal distribution.

In statistic, the information in a sample $X=\left(X_{1}, X_{2}, \ldots, X_{n}\right)$ is used to make inferences about an unknown parameter $\theta$. The inference methods are hypothesis testing, point estimation, and confidence interval estimation. The statistical hypothesis makes a statement about a population parameter. The hypothesis testing uses the sample from the population for deciding. Two complementary hypotheses in hypothesis testing are the null hypothesis and the alternative hypothesis. The point estimation uses the sample data to evaluate a single value. The point estimation is a guess of the single value as the value of the parameter. The value is called the point estimator. The point estimate is not absolutely accurate because the estimate is based on only the single random sample. For a contrasting point estimation method, the confidence interval estimation uses the sample data to calculate an interval of probable values. The confidence interval is called the confidence interval estimator. The confidence interval estimation is used rather than the point estimation because the confidence interval estimation has some guarantee of capturing the parameter. The goal of this paper is to examine the confidence interval for parameter of log-normal distribution. Confidence intervals associated with various functions of the log-normal distribution parameters have been reported by Land (1988), Zhou and Gao (1997), Zhou (1998), Joulious and Debarnot (2000), Taylor et al. (2002), Krishnamoorthy and Mathew (2003), Gupta and Li (2005), Hannig et al. (2006b), and Shen et al. (2006). In these studies, confidence intervals were considered on linear functions of the mean and variance of the log-normal distribution. These results were later extended to stochastic processes such as homogeneous log-normal (Gutiérrez et al., 2007) and non-homogeneous log-normal (Gutiérrez et al., 2003).

The coefficient of variation is defined as the standard deviation divided by the mean (Kelley, 2007). It can be used to compare several populations that have different measurement units and is widely used to measure the precision and repeatability of data in many fields. In hematology and serology, the coefficient of variation has been used for the measurement of blood samples taken from different laboratories (Tsim et al., 1991) and as a measure of precision within and between laboratories (Tian, 2005). In finance, a test of the equality of the coefficients of variation for two stocks has been used to measure risk. In medicine, the coefficient of variation has been used to compare the variability in the ratio of total/high density lipoprotein cholesterol with the variability in vessel diameter change according to diet.

In climate sciences and hydrology, the coefficient of variation has been used to describe the rainfall and can be used to compare the rainfall variability in two or more different areas (Thangjai et al., 2020a). If the difference between the rainfall in a single area and the average rainfall over several areas is high, then the rainfall is high. In statistical analysis, combine the results of several independent studies is used in climate sciences and clinical trial. If it is assumed that the samples are collected from independent log-normal populations with a common coefficient of variation but possibly with different variances, then the confidence interval for the common coefficient of variation of several log-normal populations becomes the parameter of interest. Several researchers have focused on confidence interval estimation for the coefficient of variation of a log-normal distribution. For example, Niwitpong (2013) presented confidence intervals for the coefficient of variation of a log-normal distribution with a restricted parameter space. $\mathrm{Ng}$ (2014) proposed an approach to make inference on the common coefficient of variation of log-normal populations. Simultaneous confidence intervals for the differences in the coefficients of variation of log-normal distributions were proposed by Thangjai et al. (2016) and Thangjai et al. (2020a). Moreover, Nam and Kwon (2017) and Hasan and Krishnamoorthy (2017) studied the confidence intervals for the ratio of coefficients of variation of two log-normal distributions.

Common problems in applied statistics are confidence interval estimation for the coefficient of variation and testing the equality of two or more coefficients of variation. Miller and Karson (1977) proposed a test for the equality of coefficients of variation in two normal populations. Gupta and Ma (1996) presented testing the equality of the coefficients of variation in $k$ normal populations. Fung and Tsang (1998) compared parametric and nonparametric tests and Gokpinar and Gokpinar (2015) proposed a computational approach to test the equality of the coefficients of variation of $k$ normal populations. Sangnawakij (2016) proposed two new confidence intervals for the ratio of the coefficients of variation 
of two-parameter exponential distributions. Sangnawakij and Niwitpong (2016) presented confidence interval estimation for the single coefficient of variation and the difference between two coefficients of variation in two-parameter exponential distributions.

Under many circumstances, confidence interval estimation or hypothesis testing for the common coefficient of variation based on several independent samples is of interest (Tian, 2005). Krishnamoorthy and $\mathrm{Lu}$ (2003) investigated procedures for confidence interval estimation and hypothesis testing of the common mean of several normal populations, while the problem of making inference from common populations with a common coefficient of variation of normal distributions was dealt with by Tian (2005). Tian and $\mathrm{Wu}$ (2007) proposed confidence interval estimation and hypothesis testing of the common mean of several log-normal populations using the generalized variable concept. Similarly, procedures for hypothesis testing and confidence interval estimation for the common mean of several inverse Gaussian populations were presented by Ye et al. (2010). Moreover, $\mathrm{Ng}$ (2014) constructed a confidence interval for the common coefficient of variation from several independent log-normal samples based on the GCI approach, although there was no comparison with other approaches.

The concepts of the generalized pivotal quantity (GPQ) and the GCI first proposed by Weerahandi (1993) have been applied to solve many statistical problems. For example, Krishnamoorthy and Lu (2003) presented the generalized variable and GCI approaches for inference on the common mean of several normal populations. Tian (2005) studied inferences on the common coefficient of variation of several normal populations. Moreover, Tian and $\mathrm{Wu}$ (2007) proposed the generalized variable approach and the GCI approach for inferences on the common mean of several log-normal populations. Thangjai et al. (2020b) developed confidence intervals for the common coefficient of variation of several normal populations using the GCI and adjusted GCI approaches. Hannig et al. (2006a) suggested fiducial GPQ (FGPQ) as a subclass of GPQ, with the FGCI being constructed using the FGPQ under fairly general conditions. Furthermore, FGCIs have been constructed to solve many practical problems (Hannig et al., 2006b; Chang and Huang, 2009; Kharrati-Kopaei et al. 2013; Thangjai et al., 2016). Although the FGCI approach is based on simulated data, one advantage is that it can be used to construct the confidence interval for complex parameters.

The method of variance estimates recovery (MOVER) approach was introduced by Zou and Donner (2008) and Zou et al. (2009). Several researchers have successfully used the MOVER approach to construct confidence intervals (Donner and Zou, 2012; Suwan and Niwitpong, 2013; Niwitpong and Wongkhao, 2016). The MOVER approach has the advantage of being easy to compute using the exact formula. However, the disadvantage of this approach is that one can construct it with or without the initial confidence interval for a single parameter of interest.

The computational approach proposed by Pal et al. (2007) has been used by many researchers to test the equality of several populations (e.g. Jafari and Abdollahnezhad, 2015; Jafari and Kazemi, 2017; Gokpinar and Gokpinar, 2017). As an advantage, this approach does not require explicit knowledge of the sampling distribution of the test statistic. However, it is based on simulation and numerical computations using the maximum likelihood estimate only. The Bayesian approach uses Bayes' theorem to compute and update probabilities after obtaining new data. Although it can be applied to estimate the confidence intervals for complex parameters, the disadvantages of applying it are that it requires prior information and is based on simulation.

$\mathrm{Ng}$ (2014) constructed the GCI of a log-normal distribution which used asymptotic variance and provided coverage probabilities close to the nominal confidence level of 0.95 . The confidence interval constructed by transforming the log-normal coefficient of variation to the normal coefficient of variation. However, its use was only considered for homogeneous populations. Therefore, in this study, we extend the research of $\mathrm{Ng}$ (2014) to develop four novel approaches for confidence interval estimation for the common coefficient of variation of several log-normal populations based on the FGCI, MOVER, computational approach, and Bayesian approaches. Unlike Ng (2014), we compute the confidence intervals for the common coefficient of variation directly using the log-normal coefficient of variation that depends on $\sigma^{2}$ only. Moreover, there is no previous literature on applying their methodology to PM2.5 concentration measurements. Therefore, to fill the gap, the novel approaches for the confidence interval estimation of the common coefficient of variation of log-normal distributions were proposed with considering the log-normality of PM2.5 concentration measurements. 


\section{METHODS}

Let us consider $Y$ the $\log$-normal distribution with parameters $\mu_{Y}$ and $\sigma_{Y}^{2}$. It is well known that $X=\log (Y)$ follows a normal distribution with mean $\mu$ and variance $\sigma^{2}$, whereas the mean and variance of $Y$ are given by

$$
\mu_{Y}=\exp \left(\mu+\left(\sigma^{2} / 2\right)\right)
$$

and

$$
\sigma_{Y}^{2}=\left(\exp \left(\sigma^{2}\right)-1\right) \cdot\left(\exp \left(2 \mu+\sigma^{2}\right)\right)
$$

respectively. From Equation (1) and Equation (2), the coefficient of variation of $Y$ is given by

$$
\theta=\sqrt{\exp \left(\sigma^{2}\right)-1}
$$

From Equation (3), it is seen that the coefficient of variation of the log-normal distribution depends on parameter $\sigma^{2}$ only, whereas the coefficient of variation of the normal distribution depends on $\mu$ and $\sigma^{2}$. And the next result provides a useful approximation for the variance of an estimator of $\theta$.

Let $\hat{\theta}=\sqrt{\exp \left(S^{2}\right)-1}$ be an estimator of $\theta$. Following the Niwitpong (2013) and Hasan and Krishnamoorthy (2017), the variance of $\hat{\theta}$ is

$$
\operatorname{Var}(\hat{\theta}) \approx \frac{\sigma^{4} \exp \left(2 \sigma^{2}\right)}{2(n-1) \cdot\left(\exp \left(\sigma^{2}\right)-1\right)} .
$$

Suppose that random samples are taken from $k$ log-normal distributions, $Y_{i}=\left(Y_{i 1}, Y_{i 2}, \ldots, Y_{i n_{i}}\right) \sim$ $L N\left(\mu_{i}, \sigma_{i}^{2}\right)$, where $i=1,2, \ldots, k$. Let $\theta_{i}=\sqrt{\exp \left(\sigma_{i}^{2}\right)-1}$ be the coefficient of variation for $i=1,2, \ldots, k$. Let $X_{i}=\left(X_{i 1}, X_{i 2}, \ldots, X_{i n_{i}}\right)$ be a random variable of size $n_{i}$ from the normal distribution with $\mu_{i}$ and variance $\sigma_{i}^{2}$. Let $\hat{\mu}_{i}=\bar{X}_{i}$ and $\hat{\sigma}_{i}^{2}=S_{i}^{2}$ be estimators of $\mu_{i}$ and $\sigma_{i}^{2}$, respectively, where $\bar{X}_{i}$ and $S_{i}^{2}$ denote the mean and variance of the log-transformed sample from a log-normal distribution. The mean and variance are given by

$$
\bar{X}_{i}=\sum_{j=1}^{n_{i}} \frac{X_{i j}}{n_{i}}
$$

and

$$
S_{i}^{2}=\frac{\sum_{j=1}^{n_{i}}\left(X_{i j}-\bar{X}_{i}\right)^{2}}{n_{i}-1}
$$

where $i=1,2, \ldots, k$ and $j=1,2, \ldots, n_{i}$.

Let $\bar{x}_{i}$ and $s_{i}^{2}$ be the observed values of $\bar{X}_{i}$ and $S_{i}^{2}$, respectively. The maximum likelihood estimator of the coefficient of variation $\theta_{i}$, is also unbiased estimator, is given by

$$
\hat{\theta}_{i}=\sqrt{\exp \left(S_{i}^{2}\right)-1}
$$

where $S_{i}^{2}$ is defined in Equation (6).

\section{Fiducial generalized confidence interval}

The FGCI uses the FGPQs. The FGPQs are a subclass of the GPQs. The FGCI has correct frequentist coverage probability. For $i=1,2, \ldots, k$, let $\bar{X}_{i}$ and $S_{i}^{2}$ be the sample mean and the sample variance for log-transformed data and let $\bar{x}_{i}$ and $s_{i}^{2}$ be the observed sample mean and the observed sample variance respectively. Let $\bar{X}_{i}^{*}$ and $S_{i}^{2 *}$ be independent copies of $\bar{X}_{i}$ and $S_{i}^{2}$, respectively. Let $\bar{X}_{i}$ and $\bar{X}_{i}^{*}$ be independent and identically distributed with mean $\mu_{i}$ and variance $\sigma_{i}^{2} / n_{i}$. It is well known that

$$
\bar{X}_{i} \sim N\left(\mu_{i}, \frac{\sigma_{i}^{2}}{n_{i}}\right) \quad \text { and } \quad \bar{X}_{i}^{*} \sim N\left(\mu_{i}, \frac{\sigma_{i}^{2}}{n_{i}}\right)
$$


Furthermore, let $S_{i}^{2}$ and $S_{i}^{2 *}$ be independent and identically distributed. Then

$$
\frac{\left(n_{i}-1\right) S_{i}^{2}}{\sigma_{i}^{2}} \sim \chi_{n_{i}-1}^{2} \quad \text { and } \quad \frac{\left(n_{i}-1\right) S_{i}^{2 *}}{\sigma_{i}^{2}} \sim \chi_{n_{i}-1}^{2},
$$

$$
R_{\sigma_{i}^{2}}=\frac{S_{i}^{2}}{S_{i}^{2 *}} \sigma_{i}^{2}
$$

Therefore, the FGPQ for $\theta_{i}$ based on the FGPQ for $\sigma_{i}^{2}$ is given by

$$
R_{\theta_{i}}=\sqrt{\exp \left(R_{\sigma_{i}^{2}}\right)-1 .}
$$

The FGPQ of $\theta_{i}$ in Equation (12) satisfies two conditions defined in Definition of Hannig et al. (2006a, 2006b). The definition of Hannig et al. (2006a, 2006b) has two conditions such as the distribution of the GPQ is free of all unknown parameters and the observed value of the GPQ is the parameter of interest. From Equation (4), the variance of $\hat{\theta}_{i}$ is provided by

$$
\operatorname{Var}\left(\hat{\theta}_{i}\right) \approx \frac{\sigma_{i}^{4} \exp \left(2 \sigma_{i}^{2}\right)}{2\left(n_{i}-1\right) \cdot\left(\exp \left(\sigma_{i}^{2}\right)-1\right)} .
$$

The FGPQ of $\operatorname{Var}\left(\hat{\theta}_{i}\right)$ is given by

$$
R_{\operatorname{Var}\left(\hat{\theta}_{i}\right)}=\frac{R_{\sigma_{i}^{2}}^{2} \exp \left(2 R_{\sigma_{i}^{2}}\right)}{2\left(n_{i}-1\right) \cdot\left(\exp \left(R_{\sigma_{i}^{2}}\right)-1\right)} .
$$

The FGPQ for the common coefficient of variation $\theta$ is a weighted average of the FGPQ $R_{\theta_{i}}$ based on $k$ individual sample. Therefore, the FGPQ is given by

$$
R_{\theta}=\sum_{i=1}^{k} \frac{R_{\theta_{i}}}{R_{\operatorname{Var}\left(\hat{\theta}_{i}\right)}} / \sum_{i=1}^{k} \frac{1}{R_{\operatorname{Var}\left(\hat{\theta}_{i}\right)}},
$$

where $R_{\theta_{i}}$ is defined in Equation (12) and $R_{\operatorname{Var}\left(\hat{\theta}_{i}\right)}$ is defined in Equation (14).

The FGPQ in Equation (12) satisfies two conditions of the definition given above. The FGCI is constructed using the quantiles of FGPQ defined in Equation (15). Therefore, the $100(1-\alpha) \%$ two-sided confidence interval for the common coefficient of variation $\theta$ based on the FGCI approach is

$$
C I_{F G C I}=\left[L_{F G C I}, U_{F G C I}\right]=\left[R_{\theta}(\alpha / 2), R_{\theta}(1-\alpha / 2)\right],
$$

where $R_{\theta}(\alpha / 2)$ and $R_{\theta}(1-\alpha / 2)$ denote the $100(\alpha / 2)$-th and $100(1-\alpha / 2)$-th percentiles of $R_{\theta}$, respectively.

The following algorithm is used to construct the FGCI:

\section{Algorithm 1}

For a given $\bar{x}_{i}$ and $s_{i}^{2}$, where $i=1,2, \ldots, k$

For $g=1$ to $m$, where $m$ is number of generalized computation

Generate $X^{*}$ and then compute $\bar{x}_{i}^{*}$ and $s_{i}^{2 *}$

Generate $\chi_{n_{i}-1}^{2}$ from chi-squared distribution with $n_{i}-1$ degrees of freedom

Compute $R_{\sigma_{i}^{2}}$ from Equation (11)

Compute $R_{\theta_{i}}$ from Equation (12)

Compute $R_{\operatorname{Var}\left(\hat{\theta}_{i}\right)}$ from Equation (14)

Compute $R_{\theta}$ from Equation (15)

End $g$ loop

Compute $R_{\theta}(\alpha / 2)$ and $R_{\theta}(1-\alpha / 2)$ from Equation (16) 
Method of variance estimates recovery confidence interval

Zou and Donner (2008) and Zou et al. (2009) proposed the MOVER approach to construct the confidence interval for the sum of two parameters. For $i=1,2$, let $\theta_{1}$ and $\theta_{2}$ be the parameters of interest. Let $L$ and $U$ be the lower limit and upper limit of the confidence interval for $\theta_{1}+\theta_{2}$. Moreover, let $\hat{\theta}_{1}$ and $\hat{\theta}_{2}$ be the estimators of $\theta_{1}$ and $\theta_{2}$, respectively. The central limit theorem and the assumption of independence between the point estimates $\hat{\theta}_{1}$ and $\hat{\theta}_{2}$ are used. Therefore, the lower limit $L$ is

$$
L=\hat{\theta}_{1}+\hat{\theta}_{2}-z_{\alpha / 2} \sqrt{\widehat{\operatorname{Var}}\left(\hat{\theta}_{1}\right)+\widehat{\operatorname{Var}}\left(\hat{\theta}_{2}\right)},
$$

where $z_{\alpha / 2}$ is the $100(\alpha / 2)$-th percentile of the standard normal distribution.

Let $l_{i}$ and $u_{i}$ be the lower limit and upper limit of the confidence interval for $\theta_{i}$, where $i=1,2$. The lower limit $L$ must be closer to $l_{1}+l_{2}$ than to $\hat{\theta}_{1}+\hat{\theta}_{2}$. The variance estimate for $\hat{\theta}_{i}$ at $\theta_{i}=l_{i}$ is defined by

$$
\widehat{\operatorname{Var}}\left(\hat{\theta}_{l_{i}}\right)=\frac{\left(\hat{\theta}_{i}-l_{i}\right)^{2}}{z_{\alpha / 2}^{2}} .
$$

Substituting back into Equation (17) as follows

$$
L=\hat{\theta}_{1}+\hat{\theta}_{2}-\sqrt{\left(\hat{\theta}_{1}-l_{1}\right)^{2}+\left(\hat{\theta}_{2}-l_{2}\right)^{2}}
$$

Similarly, the variance estimate for $\hat{\theta}_{i}$ at $\theta_{i}=u_{i}$ is defined by

$$
\widehat{\operatorname{Var}}\left(\hat{\theta}_{u_{i}}\right)=\frac{\left(u_{i}-\hat{\theta}_{i}\right)^{2}}{z_{\alpha / 2}^{2}} .
$$

The upper limit $U$ is

$$
U=\hat{\theta}_{1}+\hat{\theta}_{2}+\sqrt{\left(u_{1}-\hat{\theta}_{1}\right)^{2}+\left(u_{2}-\hat{\theta}_{2}\right)^{2}} .
$$

Therefore, the variance estimate for $\hat{\theta}_{i}$ at $\theta_{i}=l_{i}$ and $\theta_{i}=u_{i}$ is defined by

$$
\widehat{\operatorname{Var}}\left(\hat{\theta}_{i}\right)=\frac{1}{2}\left(\frac{\left(\hat{\theta}_{i}-l_{i}\right)^{2}}{z_{\alpha / 2}^{2}}+\frac{\left(u_{i}-\hat{\theta}_{i}\right)^{2}}{z_{\alpha / 2}^{2}}\right),
$$

where $i=1,2$.

In this paper, the $k$ parameters of interest are $\theta_{1}, \theta_{2}, \ldots, \theta_{k}$. The concepts of Zou and Donner (2008) and Zou et al. (2009) are motivated for constructing the confidence interval for $\theta_{1}+\theta_{2}+\ldots+\theta_{k}$ are

$$
L=\hat{\theta}_{1}+\hat{\theta}_{2}+\ldots+\hat{\theta}_{k}-\sqrt{\left(\hat{\theta}_{1}-l_{1}\right)^{2}+\left(\hat{\theta}_{2}-l_{2}\right)^{2}+\ldots+\left(\hat{\theta}_{k}-l_{k}\right)^{2}}
$$

and

$$
U=\hat{\theta}_{1}+\hat{\theta}_{2}+\ldots+\hat{\theta}_{k}+\sqrt{\left(u_{1}-\hat{\theta}_{1}\right)^{2}+\left(u_{2}-\hat{\theta}_{2}\right)^{2}+\ldots+\left(u_{k}-\hat{\theta}_{k}\right)^{2}},
$$

where $\left(l_{1}, u_{1}\right),\left(l_{2}, u_{2}\right), \ldots,\left(l_{k}, u_{k}\right)$ contain the parameter values for $\theta_{1}, \theta_{2}, \ldots, \theta_{k}$, respectively.

According to Graybill and Deal (1959), the common coefficient of variation $\theta$ is weighted average of the coefficient of variation $\hat{\theta}_{i}$ based on $k$ individual samples. Therefore, the common coefficient of variation is defined by

$$
\hat{\theta}=\sum_{i=1}^{k} \frac{\hat{\theta}_{i}}{\widehat{\operatorname{Var}}\left(\hat{\theta}_{i}\right)} / \sum_{i=1}^{k} \frac{1}{\widehat{\operatorname{Var}}\left(\hat{\theta}_{i}\right)},
$$

where $\hat{\theta}_{i}=\sqrt{\exp \left(S_{i}^{2}\right)-1}$ and $\widehat{\operatorname{Var}}\left(\hat{\theta}_{i}\right)$ is defined in Equation (22). 
Applying Krishnamoorthy and Oral (2017), the lower limit and upper limit of the confidence interval for the common coefficient of variation $\theta$ are defined by

$$
L_{M O V E R}=\hat{\theta}-\sqrt{\sum_{i=1}^{k} \frac{\left(\hat{\theta}_{i}-l_{i}\right)^{2}}{\left(\widehat{\operatorname{Var}}\left(\hat{\theta}_{l_{i}}\right)\right)^{2}} / \sum_{i=1}^{k} \frac{1}{\left(\widehat{\operatorname{Var}}\left(\hat{\theta}_{l_{i}}\right)\right)^{2}}}
$$

and

$$
U_{M O V E R}=\hat{\theta}+\sqrt{\sum_{i=1}^{k} \frac{\left(u_{i}-\hat{\theta}_{i}\right)^{2}}{\left(\widehat{\operatorname{Var}}\left(\hat{\theta}_{u_{i}}\right)\right)^{2}} / \sum_{i=1}^{k} \frac{1}{\left(\widehat{\operatorname{Var}}\left(\hat{\theta}_{u_{i}}\right)\right)^{2}}}
$$

where $\hat{\theta}$ is defined in Equation (25).

According to Niwitpong (2013), for $i=1,2, \ldots, k$, the confidence interval for coefficient of variation of log-normal distribution based on the $i$-th sample is given by

$$
\left[l_{i}, u_{i}\right]=\left[\sqrt{\exp \left(\frac{\left(n_{i}-1\right) S_{i}^{2}}{\chi_{\left(n_{i}-1\right),(1-\alpha / 2)}^{2}}\right)-1}, \sqrt{\exp \left(\frac{\left(n_{i}-1\right) S_{i}^{2}}{\chi_{\left(n_{i}-1\right),(\alpha / 2)}^{2}}\right)-1},\right.
$$

where $\chi_{\left(n_{i}-1\right),(1-\alpha / 2)}^{2}$ and $\chi_{\left(n_{i}-1\right),(\alpha / 2)}^{2}$ denote the $100(1-\alpha / 2)$-th and $100(\alpha / 2)$-th percentiles of the chi-squared distribution with $n_{i}-1$ degrees of freedom.

Therefore, the $100(1-\alpha) \%$ two-sided confidence interval for the common coefficient of variation $\theta$ based on MOVER approach is

$$
C I_{M O V E R}=\left[L_{M O V E R}, U_{M O V E R}\right],
$$

where $L_{M O V E R}$ is defined in Equation (26), $U_{M O V E R}$ is defined in Equation (27), and $l_{i}$ and $u_{i}$ are defined in Equation (28).

\section{Computational confidence interval}

Theorem 1: Let $Y_{i}=\left(Y_{i 1}, Y_{i 2}, \ldots, Y_{i n_{i}}\right)$ be a log-normal population with parameters $\mu_{i}$ and $\sigma_{i}^{2}$, where $i=1,2, \ldots, k$. For $i=1,2, \ldots, k$ and $j=1,2, \ldots, n_{i}$, let $X_{i j}=\log \left(Y_{i j}\right)$ be the normal distribution with mean $\mu_{i}$ and variance $\sigma_{i}^{2}$. The maximum likelihood estimators of $\mu_{i}$ and $\theta$ given by Equation (3) under $\theta_{1}=\theta_{2}=\ldots=\theta_{k}=\theta$ are given by

$$
\hat{\mu}_{i}=\bar{X}_{i}
$$

and

$$
\sum_{i=1}^{k} \frac{n_{i} \hat{\sigma}_{i}^{2}}{\log \left(\theta^{2}+1\right)}-\sum_{i=1}^{k} n_{i}=0
$$

Proof: The log-likelihood function of normal distribution with parameters $\mu_{i}$ and $\theta$ is given by

$$
\ln L=-\frac{1}{2} \sum_{i=1}^{k} n_{i} \log \left(2 \pi \log \left(\theta^{2}+1\right)\right)-\sum_{i=1}^{k} n_{i} \log \left(Y_{i j}\right)-\sum_{i=1}^{k} \sum_{j=1}^{n_{i}} \frac{\left(\log \left(Y_{i j}\right)-\mu_{i}\right)^{2}}{2 \log \left(\theta^{2}+1\right)}
$$

Differentiating the $\ln L$ with respect to $\mu_{i}$ and $\theta$, respectively, the maximum likelihood estimators of $\mu_{i}$ and $\theta$ are given by

$$
\hat{\mu}_{i}=\bar{X}_{i}
$$

and

$$
\sum_{i=1}^{k} \frac{n_{i} \hat{\sigma}_{i}^{2}}{\log \left(\theta^{2}+1\right)}-\sum_{i=1}^{k} n_{i}=0
$$


Hence, Theorem 1 is proved.

According to Pal et al. (2007), the computational approach uses the maximum likelihood estimates (MLEs). The common coefficient of variation based on maximum likelihood estimator is defined by

$$
\hat{\theta}_{M L}=\sum_{i=1}^{k} \frac{\hat{\theta}_{i}}{\widehat{\operatorname{Var}}\left(\hat{\theta}_{i}\right)} / \sum_{i=1}^{k} \frac{1}{\widehat{\operatorname{Var}}\left(\hat{\theta}_{i}\right)},
$$

where $\hat{\theta}_{i}=\sqrt{\exp \left(S_{i}^{2}\right)-1}$ and $\widehat{\operatorname{Var}}\left(\hat{\theta}_{i}\right)$ is defined in Equation (4) with $\sigma_{i}$ replaced by $s_{i}$.

The computational approach is to obtain the restricted maximum likelihood estimates (RMLEs) of parameters. The maximum likelihood estimators of $\mu_{i}$ and $\theta$ under $\theta_{1}=\theta_{2}=\ldots=\theta_{k}=\theta$ provide the RMLEs of these parameters.

Then the RMLE of $\mu_{i}$ is defined by $\hat{\mu}_{i(R M L)}=\bar{X}_{i}$. The RMLE of $\theta$ obtained iteratively from Equation (31) by using bisection method. The $\theta$ converge to the RMLE denoted as $\hat{\theta}_{R M L}$.

Data replication from $f\left(x \mid \hat{\mu}_{i(R M L)}, \hat{\theta}_{R M L}\right)$ is used to construct the confidence interval based on computational approach. Let artificial sample $X_{i(R M L)}=\left(X_{i 1(R M L)}, X_{i 2(R M L)}, \ldots, X_{i n_{i}(R M L)}\right)$ be the normal distribution with mean $\hat{\mu}_{i(R M L)}$ and variance $\hat{\sigma}_{i(R M L)}^{2}$. Let $\bar{X}_{i(R M L)}$ and $S_{i(R M L)}^{2}$ be the mean and variance of the log-transformed sample from a log-normal distribution for the $i$-th artificial sample and let $\bar{x}_{i(R M L)}$ and $s_{i(R M L)}^{2}$ be observed sample mean and observed sample variance, respectively.

Therefore, the common coefficient of variation based on $k$ individual samples is defined by

$$
\hat{\theta}_{R M L}=\sum_{i=1}^{k} \frac{\hat{\theta}_{i(R M L)}}{\widehat{\operatorname{Var}}\left(\hat{\theta}_{i(R M L)}\right)} / \sum_{i=1}^{k} \frac{1}{\widehat{\operatorname{Var}}\left(\hat{\theta}_{i(R M L)}\right)}
$$

where $\hat{\theta}_{i(R M L)}=\sqrt{\exp \left(S_{i(R M L)}^{2}\right)-1}$.

Therefore, the $100(1-\alpha) \%$ two-sided confidence interval for the common coefficient of variation $\theta$ based on computational approach is

$$
C I_{C A}=\left[L_{C A}, U_{C A}\right]=\left[\hat{\theta}_{R M L}(\alpha / 2), \hat{\theta}_{R M L}(1-\alpha / 2)\right],
$$

where $\hat{\theta}_{R M L}(\alpha / 2)$ and $\hat{\theta}_{R M L}(1-\alpha / 2)$ denote the $(\alpha / 2)$-th and $(1-\alpha / 2)$-th percentiles of $\hat{\theta}_{R M L}$, respectively.

The following algorithm is used to construct the computational confidence interval:

\section{Algorithm 2}

For a given $\bar{x}_{i}, s_{i}^{2}$, and $\theta$, where $i=1,2, \ldots, k$

Compute $\hat{\mu}_{i(R M L)}$ and $\hat{\theta}_{R M L}$ from Equation (30)-Equation (31)

For $g=1$ to $m$

Generate $x_{i j(R M L)}$ from $N\left(\hat{\mu}_{i(R M L)}, \sqrt{\log \left(\hat{\theta}_{R M L}^{2}+1\right)}\right)$

Compute $\bar{x}_{i(R M L)}$ and $s_{i(R M L)}^{2}$

Compute $\hat{\theta}_{R M L}$ from Equation (33)

End $g$ loop

Compute $\hat{\theta}_{R M L}(\alpha / 2)$ and $\hat{\theta}_{R M L}(1-\alpha / 2)$ from Equation (34)

\section{Bayesian confidence interval}

The FGCI approach, MOVER approach, and computational approach are the classical approach. The classical approach and the Bayesian approach are fundamentally different. In the classical approach, the parameter of interest $\theta$ is unknown, but it is fixed. In the Bayesian approach, the parameter is considered to be a quantity. The variation of the quantity is described by the prior distribution. Bayes (1763) introduced that Bayesian approach uses Bayes' theorem to update probabilities. Bayes' theorem describes the conditional probability of an event based on data. The data is prior information or beliefs about the event. The posterior distribution is combination of the likelihood function and the prior distribution. The Bayesian confidence interval is constructed based on the posterior distribution. The posterior distribution 
is a conditional distribution which is based on the observed values of the sample. The posterior distribution is used to make statements about the parameter. The parameter is considered a random quantity. The conditional posterior distribution for $\mu_{i}$ given $\sigma_{i}^{2}$ and $x_{i}$ is the normal distribution with mean $\hat{\mu}_{i}$ and variance $\sigma_{i}^{2} / n_{i}$. The distribution is defined by

$$
\mu_{i} \mid \sigma_{i}^{2}, x_{i} \sim N\left(\hat{\mu}_{i}, \sigma_{i}^{2} / n_{i}\right)
$$

The posterior distribution for $\sigma_{i}^{2}$ is inverse gamma distribution. It is defined by

$$
\sigma_{i}^{2} \mid x_{i} \sim I G\left(\left(n_{i}-1\right) / 2,\left(n_{i}-1\right) s_{i}^{2} / 2\right)
$$

The posterior distribution of coefficient of variation of log-normal distribution is

$$
\hat{\theta}_{i}=\sqrt{\exp \left(\sigma_{i}^{2}\right)-1}
$$

where $\sigma_{i}^{2}$ is defined in Equation (36).

The variance of $\hat{\theta}_{i}$ is

$$
\operatorname{Var}\left(\hat{\theta}_{i}\right) \approx \frac{\sigma_{i}^{4}\left(\exp \left(2 \sigma_{i}^{2}\right)\right)}{2\left(n_{i}-1\right)\left(\exp \left(\sigma_{i}^{2}\right)-1\right)},
$$

where $\sigma_{i}^{2}$ is defined in Equation (36).

The common coefficient of variation of log-normal distribution based on $k$ individual samples which the parameter of interest defined by

$$
\hat{\theta}_{B S}=\sum_{i=1}^{k} \frac{\hat{\theta}_{i}}{\operatorname{Var}\left(\hat{\theta}_{i}\right)} / \sum_{i=1}^{k} \frac{1}{\operatorname{Var}\left(\hat{\theta}_{i}\right)},
$$

where $\hat{\theta}_{i}$ is defined in Equation (37) and $\operatorname{Var}\left(\hat{\theta}_{i}\right)$ is defined in Equation (38).

Gelman et al. (2013) introduced the highest posterior density interval to construct the Bayesian confidence interval. Therefore, the $100(1-\alpha) \%$ two-sided confidence interval for the common coefficient of variation $\theta$ based on Bayesian approach is

$$
C I_{B S}=\left[L_{B S}, U_{B S}\right],
$$

where $L_{B S}$ and $U_{B S}$ are the lower limit and the upper limit of the shortest $100(1-\alpha) \%$ highest posterior density interval of $\hat{\theta}_{B S}$, respectively.

The following algorithm is used to construct the Bayesian confidence interval:

\section{Algorithm 3}

For a given $\bar{x}_{i}$ and $s_{i}^{2}$, where $i=1,2, \ldots, k$

For $g=1$ to $m$

Generate $\mu_{i} \mid \sigma_{i}^{2}, x_{i} \sim N\left(\hat{\mu}_{i}, \sigma_{i}^{2} / n_{i}\right)$

Generate $\sigma_{i}^{2} \mid x_{i} \sim \operatorname{IG}\left(\left(n_{i}-1\right) / 2,\left(n_{i}-1\right) s_{i}^{2} / 2\right)$

Compute $\theta_{i}$ from Equation (37)

Compute $\operatorname{Var}\left(\hat{\theta}_{i}\right)$ from Equation (38)

Compute $\theta_{B S}$ from Equation (39)

End $g$ loop

Compute $L_{B S}$ and $U_{B S}$

\section{RESULTS}

A simulation study was performed to evaluate the coverage probabilities and average lengths of the FGCI $\left(C I_{F G C I}\right)$, MOVER $\left(C I_{M O V E R}\right)$, computational $\left(C I_{C A}\right)$, and Bayesian confidence intervals $\left(C I_{B S}\right)$. The confidence intervals were compared by measuring their coverage probabilities and average lengths and, in each case, the one with a coverage probability closest to the nominal confidence level $(1-\alpha)$ and with the shortest average length was chosen as the most appropriate. 
In this simulation study, the nominal confidence level was chosen as 0.95 . The sample cases used were $k=3$ and $k=6$ with sample sizes $n_{1}, n_{2}, \ldots, n_{k}$, as in Tables 1 and 2. Following Tian and Wu (2007) and $\mathrm{Ng}$ (2014), the coefficient of variation of log-normal distribution, which equals $\sqrt{\exp \left(\sigma^{2}\right)-1}$, is chosen in the range from $0.05-2.00$. Since the coefficient of variation of a log-normal distribution is independent of $\mu$, the population means of the normal data within each sample were given the same value $\mu_{1}=\mu_{2}=\ldots=\mu_{k}=\mu=1$ to simplify matters, and the population standard deviations $\sigma_{1}, \sigma_{2}, \ldots, \sigma_{k}$ are as in Tables 1 and 2. For each parameter setting, 5000 random samples were generated by applying Algorithm 4, and thus $1000 R_{\theta}, 1000 \hat{\theta}_{R M L}$, and $1000 \theta_{B S}$ were simulated by applying Algorithms 1,2 , and 3 , respectively, for each of the random samples.

The following algorithm is used to estimate the coverage probability and average length:

\section{Algorithm 4}

For a given $\left(n_{1}, n_{2}, \ldots, n_{k}\right),\left(\mu_{1}, \mu_{2}, \ldots, \mu_{k}\right),\left(\sigma_{1}, \sigma_{2}, \ldots, \sigma_{k}\right)$ and $\theta$

For $h=1$ to $M$

Generate $x_{i j}$ from $N\left(\mu_{i}, \sigma_{i}^{2}\right)$, where $i=1,2, \ldots, k$ and $j=1,2, \ldots, n_{i}$

Calculate $\bar{x}_{i}$ and $s_{i}^{2}$

Construct $\left[L_{F G C I(h)}, U_{F G C I(h)}\right]$

Construct $\left[L_{M O V E R(h)}, U_{M O V E R(h)}\right]$

Construct $\left[L_{C A(h)}, U_{C A(h)}\right]$

Construct $\left[L_{B S(h)}, U_{B S(h)}\right]$

Record whether or not all the values of $\theta$ fall in their corresponding confidence intervals

Compute $U_{(h)}-L_{(h)}$

End $h$ loop

Compute the coverage probability and the average length for each confidence interval

Tables 1 and 2 report the coverage probabilities and average lengths for $k=3$ and $k=6$, respectively. From Table 1, the simulation results indicate that for all sample sizes, the FGCI approach provided the best coverage probabilities whereas the MOVER confidence interval attained coverage probabilities under the nominal confidence level of 0.95 . Furthermore, the coverage probabilities of the MOVER confidence interval decreased when the sample sizes increased. The computational confidence interval achieved coverage probabilities under the nominal confidence level of 0.95 , which became closer to it as the sample sizes increased. The coverage probabilities of the Bayesian confidence interval performed well when the population standard deviations were $(0.05,0.10,0.15)$ whereas they were less than the nominal confidence level of 0.95 when the population standard deviations were $(0.50,1.00,1.00)$.

From Table 2, it can be seen that the coverage probabilities of the FGCI and the computational confidence interval were less than the nominal confidence level of 0.95 when the sample sizes were small. For large sample sizes, the coverage probabilities of the FGCI and the computational confidence interval were close to the nominal confidence level of 0.95, with those of the FGCIs being closer. The coverage probabilities of the MOVER confidence interval were greater than the nominal confidence level of 0.95 for small sample sizes and became close to 1.00 when the sample sizes increased, thereby showing conservative behavior when the sample case $(k)$ was large and the sample sizes were large. Therefore, the MOVER approach can be considered as an alternative to estimate the confidence interval for the common coefficient of variation of log-normal distributions when the sample case $(k=6)$ is large and the sample sizes are small. The coverage probabilities of the Bayesian confidence interval were less than the nominal confidence level of 0.95 when the sample sizes were small and were close to the nominal confidence level of 0.95 when the sample sizes were large. The average lengths of the Bayesian confidence interval were shorter than those of the others.

As the sample case $(k)$ increased, the coverage probabilities of the FGCI and the computational confidence interval tended to decrease because the common coefficient of variation $\theta$ is based on the variance of the coefficient of variation $\hat{\theta}_{i}$ for the $k$ individual samples. Herein, we present only the results of $\mu=1$ because they tended toward the same direction regardless of the value of $\mu$. In all cases, the coverage probabilities were affected by large $\sigma$ values because the coefficient of variation of log-normal distributions $\theta=\sqrt{\exp \left(\sigma^{2}\right)-1}$ depends on parameter $\sigma$ only. 
Table 1. The coverage probabilities and average lengths of $95 \%$ two-sided confidence intervals for the common coefficient of variation of several log-normal populations: 3 sample cases.

\begin{tabular}{|c|c|c|c|c|c|}
\hline \multirow{2}{*}{$\left(n_{1}, n_{2}, n_{3}\right)$} & \multirow{2}{*}{$\left(\sigma_{1}, \sigma_{2}, \sigma_{3}\right)$} & \multicolumn{4}{|c|}{ Coverage probability (Average length) } \\
\hline & & $C I_{F G C I}$ & $C I_{M O V E R}$ & $C I_{C A}$ & $C I_{B S}$ \\
\hline \multirow[t]{4}{*}{$(30,30,30)$} & $(0.05,0.10,0.15)$ & 0.9514 & 0.9238 & 0.9188 & 0.9400 \\
\hline & & $(0.0317)$ & $(0.0306)$ & $(0.0305)$ & $(0.0309)$ \\
\hline & $(0.50,1.00,1.00)$ & 0.9496 & 0.9150 & $0.9246)$ & 0.9394 \\
\hline & & $(0.3922)$ & $(0.3672)$ & $(0.3628$ & $(0.3781)$ \\
\hline \multirow[t]{4}{*}{$(50,50,50)$} & $(0.05,0.10,0.15)$ & 0.9492 & 0.9234 & 0.9244 & 0.9408 \\
\hline & & $(0.0246)$ & $(0.0230)$ & $(0.0240)$ & $(0.0241)$ \\
\hline & $(0.50,1.00,1.00)$ & 0.9504 & 0.9054 & 0.9372 & 0.9442 \\
\hline & & $(0.3060)$ & $(0.2707)$ & $(0.2909)$ & $(0.2976)$ \\
\hline \multirow[t]{4}{*}{$(30,50,100)$} & $(0.05,0.10,0.15)$ & 0.9530 & 0.9160 & 0.9200 & 0.9426 \\
\hline & & $(0.0331)$ & $(0.0312)$ & $(0.0329)$ & $(0.0325)$ \\
\hline & $(0.50,1.00,1.00)$ & 0.9498 & 0.8808 & 0.9186 & 0.9360 \\
\hline & & $(0.4259)$ & $(0.3831)$ & $(0.4055)$ & $(0.4147)$ \\
\hline \multirow[t]{4}{*}{$(50,100,200)$} & $(0.05,0.10,0.15)$ & 0.9528 & 0.9222 & 0.9358 & 0.9430 \\
\hline & & $(0.0251)$ & $(0.0234)$ & $(0.0252)$ & $(0.0247)$ \\
\hline & $(0.50,1.00,1.00)$ & 0.9492 & 0.8722 & 0.9314 & 0.9410 \\
\hline & & $(0.3357)$ & $(0.2875)$ & $(0.3272)$ & $(0.3291)$ \\
\hline \multirow[t]{4}{*}{$(100,100,100)$} & $(0.05,0.10,0.15)$ & 0.9502 & 0.9214 & 0.9388 & 0.9442 \\
\hline & & $(0.0174)$ & $(0.0159)$ & $(0.0172)$ & $(0.0171)$ \\
\hline & $(0.50,1.00,1.00)$ & 0.9478 & 0.8952 & 0.9398 & 0.9394 \\
\hline & & $(0.2176)$ & $(0.1852)$ & $(0.2117)$ & $(0.2129)$ \\
\hline \multirow[t]{4}{*}{$(200,200,200)$} & $(0.05,0.10,0.15)$ & 0.9446 & 0.9178 & 0.9396 & 0.9404 \\
\hline & & $(0.0123)$ & $(0.0111)$ & $(0.0122)$ & $(0.0121)$ \\
\hline & $(0.50,1.00,1.00)$ & 0.9488 & 0.8944 & 0.9456 & 0.9434 \\
\hline & & $(0.1542)$ & $(0.1288)$ & $(0.1520)$ & $(0.1513)$ \\
\hline \multirow[t]{4}{*}{$(500,500,500)$} & $(0.05,0.10,0.15)$ & 0.9482 & 0.9200 & 0.9444 & 0.9420 \\
\hline & & $(0.0078)$ & $(0.0070)$ & (0.0078) & $(0.0077)$ \\
\hline & $(0.50,1.00,1.00)$ & 0.9480 & 0.8940 & 0.9470 & 0.9430 \\
\hline & & $(0.0976)$ & $(0.0807)$ & (0.0971) & $(0.0961)$ \\
\hline \multirow[t]{4}{*}{$(1000,1000,1000)$} & $(0.05,0.10,0.15)$ & 0.9428 & 0.9162 & 0.9446 & 0.9390 \\
\hline & & $(0.0055)$ & $(0.0049)$ & (0.0055) & $(0.0054)$ \\
\hline & $(0.50,1.00,1.00)$ & 0.9574 & 0.9020 & 0.9552 & 0.9524 \\
\hline & & $(0.0691)$ & $(0.0569)$ & $(0.0689)$ & $(0.0681)$ \\
\hline
\end{tabular}

\section{An empirical application}

The rainfall has been the seasonal problem in Thailand. Rainy season in Thailand is between May and October. The daily rainfall appears on 17 June 2020. Real data example of rainfall data is used to illustrate the FGCI, MOVER, computational, and Bayesian approaches. All data were reported by the Thai Meteorological Department (https://www.tmd.go.th/climate/climate.php).

The rainfall data on 17 June 2020 in Northern, Northeastern, Central, Eastern, and Southern regions are reported in Dataset 1, and histogram and normal QQ-plots are presented in Figures 1 and 2, respectively. The data sets consist of 30 measurements in Northern region, 31 measurements in Northeastern region, 21 measurements in Central region, 16 measurements in Eastern region, and 27 measurements in Southern region. The statistics is summarized in Table 3. The Shapiro-Wilk normality test is used to check the assumption that the log-data is normal distribution. The Shapiro-Wilk normality test with p-values 0.2176 , 0.4981, 0.0009, 0.0128, and 0.2127 for Northern, Northeastern, Central, Eastern, and Southern regions, respectively. From p-values, the results show that the rainfall of the three regions follow log-normal distributions such as Northern, Northeastern, and Southern regions. The data of Northern, Northeastern, and Southern regions were used to construct the confidence interval for the common coefficient of variation based on the four approaches. The true common coefficient of variation was 1.2084. The point estimators of the common coefficient of variation based on the FGCI, MOVER, CA, and Bayesian approaches 

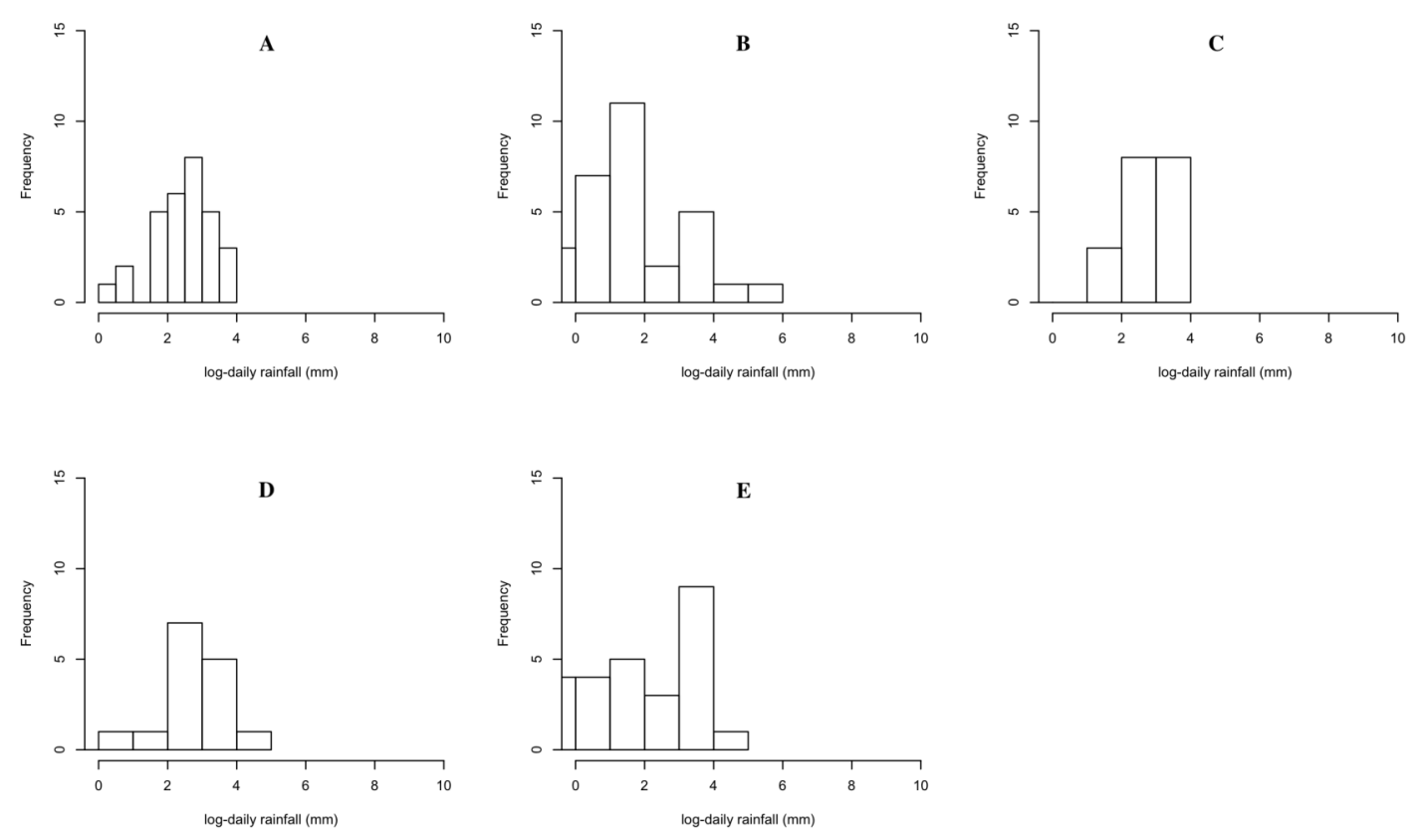

were 1.2776,1.1486, 1.2273, and 1.2669. The FGCI, MOVER, computational, and Bayesian confidence intervals were [0.8380,2.0301] with an interval length of 1.1921, [0.8460,1.8481] with an interval length of 1.0021, [0.7745,1.8663] with an interval length of 1.0918, and [0.7991,1.7704] with an interval length of 0.9713 , respectively. Hence, the length of the Bayesian confidence interval was shorter than those of the others, and so was more accurate.

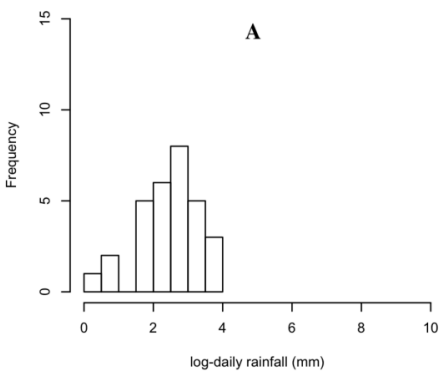

Figure 1. Histogram of rainfall data in (A) Northern (B) Northeastern (C) Central (D) Eastern (E) Southern regions.

\section{DISCUSSION}

As a limitation of this study, the coefficient of variation of log-normal distribution can be computed directly from log-normal data and the data transformed using the log function. The coefficients of variation based directly on log-normal data make it easy to construct the confidence interval for the common coefficient of variation of log-normal distributions because the coefficient of variation of a log-normal distribution is based on $\sigma^{2}$ only. However, for a greater number of samples, i.e. $n=500$ and a large $\sigma^{2}$, i.e. $\sigma^{2}=10$, the coverage probabilities of all of the proposed confidence intervals cannot be computed (the simulation results are not reported here). The coefficients of variation based on transformed data using the log function make it more difficult to estimate the confidence interval for the common coefficient of variation of log-normal distributions because the coefficient of variation of a normal distribution is based on both $\mu$ and $\sigma^{2}$.

The MOVER approach makes it possible to compute the confidence interval online because this approach requires a simple formula to construct the confidence interval. Since the FGCI, computational, and Bayesian approaches are based on simulation techniques, it is not possible to compute the confidence intervals for them online.

As a final note, the results of the computational approach did not perform well for the confidence interval estimation for the common coefficient of variation of log-normal distributions. However, Thangjai et al. (2020b) reported that it performs well for constructing the confidence interval for the common coefficient of variation of normal distributions when the sample case is large. 
A

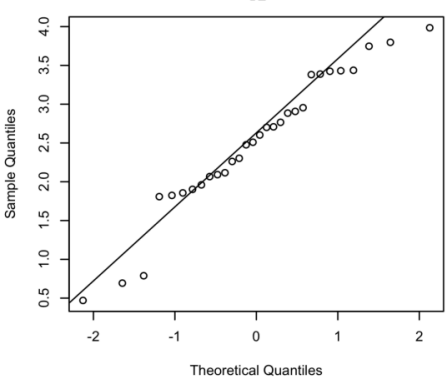

D

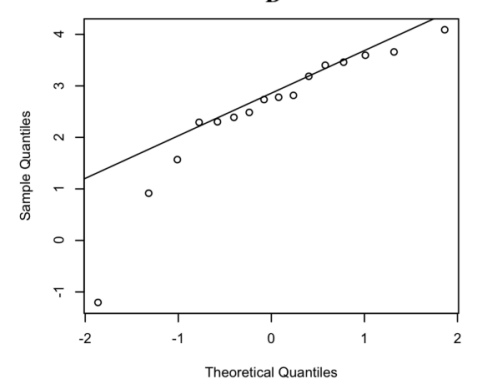

B

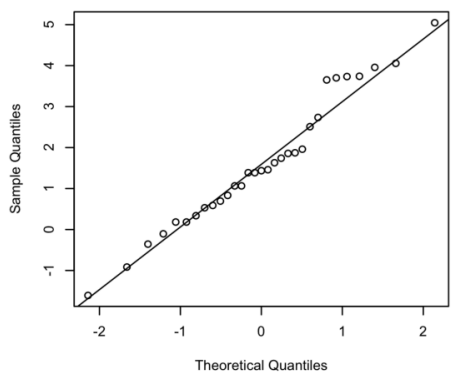

E

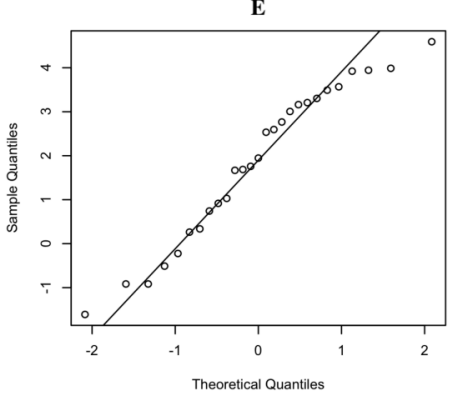

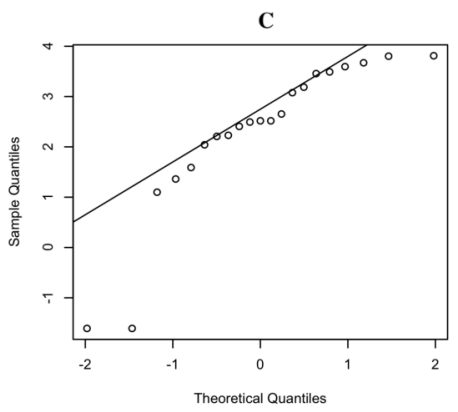

Figure 2. QQ-plot of log- rainfall data in (A) Northern (B) Northeastern (C) Central (D) Eastern (E) Southern regions.

\section{CONCLUSIONS}

The results in Tables 1 and 2 indicate that the FGCI approach provided much better confidence interval estimates than the other approaches in terms of coverage probability for almost all sample cases $(k)$ and sample sizes $(n)$, except that the MOVER approach provided the best confidence interval estimates when the sample case was equal to six populations $(k=6)$ and the sample sizes were small $\left(n_{i}<50\right)$. Moreover, the FGCI approach was the best for constructing the confidence interval for all sample sizes $(n)$ when the sample case is small $(k=3)$, for which the coverage probability of the FGCI approach was stable around the nominal confidence level of 0.95 . For large sample cases $(k=6)$, the FGCI approach performed well for confidence interval estimation when the sample sizes were large. Furthermore, the FGPQ for the FGCI approach is not dependent on the population variances $\left(\sigma_{i}\right)$. The results are similar to those of Hannig et al. (2006b), Chang and Huang (2009), Kharrati-Kopaei et al. (2013), and Thangjai et al. (2016). Note that the GCIs based on $\mathrm{Ng}$ (2014) had coverage probabilities close to 1.00 when the sample size was small and close to the nominal confidence level of 0.95 when the sample size was large. Therefore, the GCIs based on $\mathrm{Ng}$ (2014) are rather conservative for small sample sizes.

\section{REFERENCES}

Bayes T. 1763. An essay towards solving a problem in the doctrine of chances. Philosophical Transactions of the Royal Society of London 53:370-418.

Casella G, Berger RL. 2002. Statistical Inference. California: Duxbury Press.

Chang YP, Huang WT. 2009. Simultaneous fiducial generalized confidence intervals for all pairwise comparisons of means. International Journal of Information and Management Sciences 20:459-467.

Donner A, Zou GY. 2012. Closed-form confidence intervals for function of the normal standard deviation. Statistical Methods in Medical Research 21:347-359.

Fung WK, Tsang TS. 1998. A simulation study comparing tests for the equality of coefficients of variations. Statistics in Medicine 17:2003-2014.

Gelman A, Carlin JB, Stern HS, Dunson DB, Vehtari A, Rubin DB. 2013. Bayesian data analysis. 
Florida: Chapman and Hall/CRC.

Gokpinar F, Gokpinar E. 2015. A computational approach for testing equality of coefficients of variation in k normal populations. Hacettepe Journal of Mathematics and Statistics 44:1197-1213.

Gokpinar F, Gokpinar E. 2017. Testing the equality of several lognormal means based on a computational approach. Communications in Statistics - Simulation and Computation 46:1998-2010.

Graybill FA, Deal RB. 1959. Combining unbiased estimators. Biometrics 15:543-550.

Gupta RC, Li X. 2005. Statistical inference for the common mean of two log-normal distributions and some applications in reliability. Computational Statistics and Data Analysis 50:3141-3164.

Gupta RC, Ma S. 1996. Testing the equality of the coefficients of variation in $k$ normal populations. Communications in Statistics - Theory and Methods 25:115-132.

Gutiérrez R, Rico N, Román P, Torres F. 2007. Approximate and generalized confidence bands for the mean and mode functions of the lognormal diffusion process. Computational Statistics and Data Analysis 51:4038-4053.

Gutiérrez R, Román P, Romero D, Torres F. 2003. Forecasting for the univariate lognormal diffusion process with exogenous factors. Cybernetics and Systems 34:709-724.

Hannig J, Iyer H, Patterson P. 2006a. Fiducial generalized confidence intervals. Journal of the American Statistical Association 101:254-269.

Hannig J, Lidong E, Abdel-Karim A, Iyer H. 2006b. Simultaneous fiducial generalized confidence intervals for ratios of means of lognormal distributions. Austrian Journal of Statistics 35:261-269.

Hasan MS, Krishnamoorthy K. 2017. Improved confidence intervals for the ratio of coefficients of variation of two lognormal distributions. Journal of Statistical Theory and Applications 16:345-353.

Jafari AA, Abdollahnezhad K. 2015. Inferences on the means of two log-Normal distributions: A computational approach test. Communications in Statistics - Simulation and Computation 44:1659-1672. Jafari AA, Kazemi MR. 2017. Computational approach test for inference about several correlation coefficients: Equality and common. Communications in Statistics - Simulation and Computation 46:20432056.

Joulious SA, Debarnot CAM. 2000. Why are pharmacokinetics data summarize by arithmetic means?. Journal of Biopharmaceutical Statistics 10:55-71.

Kharrati-Kopaei M, Malekzadeh A, Sadooghi-Alvandi M. 2013. Simultaneous fiducial generalized confidence intervals for the successive differences of exponential location parameters under heteroscedasticity. Statistics and Probability Letters 83:1547-1552.

Kelley K. 2007. Sample size planning for the coefficient of variation from the accuracy in parameter estimation approach. Behavior Research Methods 39:755-766.

Krishnamoorthy K, Lu Y. 2003. Inference on the common means of several normal populations based on the generalized variable method. Biometrics 59:237-247.

Krishnamoorthy K, Mathew T. 2003. Inferences on the means of lognormal distributions using generalized p-values and generalized confidence intervals. Journal of Statistical Planning and Inference 115:103-121.

Krishnamoorthy K, Oral E. 2017. Standardized likelihood ratio test for comparing several log-normal means and confidence interval for the common mean. Statistical Methods in Medical Research 26:29192937.

Land CE. 1988. Hypothesis tests and interval estimates. In Crow, E.L. and Shimizu, K. (Eds.). Lognormal distributions: Theory and applications. New York: Marcel Dekker.

Miller EC, Karson MJ. 1977. Testing equality of two coefficients of variation. Proceedings of the Business and Economics Section Part I:278-283.

Nam JM, Kwon D. 2017. Inference on the ratio of two coefficients of variation of two lognormal distributions. Communications in Statistics-Theory and Methods 46: 8575-8587.

Niwitpong S. 2013. Confidence intervals for coefficient of variation of lognormal distribution with restricted parameter space. Applied Mathematical Sciences 7:3805-3810.

Niwitpong, S. and Wongkhao, A. 2016. Confidence intervals for the difference between inverse of normal means. Advances and Applications in Statistics 48:337-347.

Ng CK. 2014. Inference on the common coefficient of variation when populations are lognormal: A simulation-based approach. Journal of Statistics: Advances in Theory and Applications 11:117-134.

Pal N, Lim WK, and Ling CH. 2007. A computational approach to statistical inferences. Journal of Applied Probability and Statistics 2:13-35. 
Rumburg B, Alldredge R, Claiborn C. 2001. Statistical distributions of particulate matter and the error associated with sampling frequency. Atmospheric Environment 35:2907-2920.

Sangnawakij P, Niwitpong S. 2016. Confidence intervals for coefficients of variation in two-parameter exponential distributions. Communications in Statistics - Simulation and Computation 46:6618-6630.

Sangnawakij P, Niwitpong S, Niwitpong S. 2016. Confidence intervals for the ratio of coefficients of variation in the two-parameter exponential distributions. Lecture Notes in Artificial Intelligence 9978:542551.

Shen H, Brown L, Hui Z. 2006. Efficient estimation of log-normal means with application to pharmacokinetics data. Statistics in Medicine 25:3023-3038.

Suwan S, Niwitpong S. 2013. Estimated variance ratio confidence interval of nonnormal distributions. Far East Journal of Mathematical Sciences 4:339-350.

Taylor DJ, Kupper LL, Muller KE. 2002. Improved approximate confidence intervals for the mean of a log-normal variable. Statistics in Medicine 21:1443-1459.

Tian L. 2005. Inferences on the common coefficient of variation. Statistics in Medicine 24:2213-2220.

Tian L, Wu J. 2007. Inferences on the common mean of several log-normal populations: The generalized variable approach. Biometrical Journal 49:944-951.

Thangjai W, Niwitpong S, Niwitpong S. 2016. Simultaneous fiducial generalized confidence intervals for all differences of coefficients of variation of log-normal distributions. Lecture Notes in Artificial Intelligence 9978:552-561.

Thangjai W, Niwitpong S, Niwitpong S. 2020a. Simultaneous confidence intervals for all differences of coefficients of variation of log-normal distributions. Hacettepe Journal of Mathematics and Statistics 48:1505-1521.

Thangjai W, Niwitpong S, Niwitpong S. 2020b. Adjusted generalized confidence intervals for the common coefficient of variation of several normal populations. Communications in Statistics-Simulation and Computation 49:194-206.

Tsim YL, Yip SP, Tsang, KS, Li KF, Wong HF. 1991. Haematogoy and Serology. In Annual Report, Honk Kong Medical Technology Association Quality Assurance Programme: 25-40.

Weerahandi S. 1993. Generalized confidence intervals. Journal of American Statistical Association 88:899-905.

Ye RD, Ma TF, Wang SG. 2010. Inferences on the common mean of several inverse Gaussian populations. Computational Statistics and Data Analysis 54:906-915.

Zou GY, Donner A. 2008. Construction of confidence limits about effect measures: A general approach. Statistics in Medicine 27:1693-1702.

Zou GY, Taleban J, Hao CY. 2009. Confidence interval estimation for lognormal data with application to health economics. Computation Statistics and Data Analysis 53: 3755-3764.

Zhou XH. 1998. Estimation of the log-normal mean. Statistics in Medicine 17:2251-2264.

Zhou XH, Gao SJ. 1997. Confidence intervals for the log-normal mean. Statistics in Medicine 16:783790 . 
Table 2. The coverage probabilities and average lengths of $95 \%$ two-sided confidence intervals for the common coefficient of variation of several log-normal populations: 6 sample cases.

\begin{tabular}{|c|c|c|c|c|c|}
\hline \multirow{2}{*}{$\left(n_{1}, n_{2}, n_{3}, n_{4}, n_{5}, n_{6}\right)$} & \multirow{2}{*}{$\left(\sigma_{1}, \sigma_{2}, \sigma_{3}, \sigma_{4}, \sigma_{5}, \sigma_{6}\right)$} & \multicolumn{4}{|c|}{ Coverage probability (Average length) } \\
\hline & & $C I_{F G C I}$ & $C I_{M O V E R}$ & $C I_{C A}$ & $C I_{B S}$ \\
\hline \multirow[t]{4}{*}{$(30,30,30,30,30,30)$} & $(0.05,0.05,0.10,0.10,0.15,0.15)$ & 0.9432 & 0.9818 & 0.8812 & 0.9328 \\
\hline & & $(0.0226)$ & $(0.0298)$ & $(0.0227)$ & $(0.0222)$ \\
\hline & $(0.50,0.50,0.50,1.00,1.00,1.00)$ & 0.9218 & 0.9838 & 0.8418 & 0.8982 \\
\hline & & $(0.2099)$ & $(0.3278)$ & $(0.2108)$ & $(0.2054)$ \\
\hline \multirow[t]{4}{*}{$(30,50,100,30,50,100)$} & $(0.05,0.05,0.10,0.10,0.15,0.15)$ & 0.9400 & 0.9800 & 0.8928 & 0.9314 \\
\hline & & $(0.0199)$ & $(0.0247)$ & $(0.0202)$ & $(0.0196)$ \\
\hline & $(0.50,0.50,0.50,1.00,1.00,1.00)$ & 0.9350 & 0.9830 & 0.8992 & 0.9224 \\
\hline & & $(0.1490)$ & $(0.2033)$ & $(0.1505)$ & $(0.1461)$ \\
\hline \multirow[t]{4}{*}{$(50,50,50,50,50,50)$} & $(0.05,0.05,0.10,0.10,0.15,0.15)$ & 0.9392 & 0.9834 & 0.9060 & 0.9342 \\
\hline & & $(0.0175)$ & $(0.0227)$ & $(0.0175)$ & $(0.0172)$ \\
\hline & $(0.50,0.50,0.50,1.00,1.00,1.00)$ & 0.9290 & 0.9890 & 0.8812 & 0.9182 \\
\hline & & $(0.1628)$ & $(0.2503)$ & $(0.1638)$ & $(0.1598)$ \\
\hline \multirow[t]{4}{*}{$(30,30,50,50,100,100)$} & $(0.05,0.05,0.10,0.10,0.15,0.15)$ & 0.9354 & 0.9808 & 0.8636 & 0.9218 \\
\hline & & $(0.0239)$ & $(0.0305)$ & $(0.0245)$ & $(0.0235)$ \\
\hline & $(0.50,0.50,0.50,1.00,1.00,1.00)$ & 0.9218 & 0.9800 & 0.8596 & 0.9048 \\
\hline & & $(0.2128)$ & $(0.2931)$ & $(0.2148)$ & $(0.2086)$ \\
\hline \multirow[t]{4}{*}{$(50,50,100,100,200,200)$} & $(0.05,0.05,0.10,0.10,0.15,0.15)$ & 0.9432 & 0.9860 & 0.9028 & 0.9360 \\
\hline & & $(0.0180)$ & $(0.0231)$ & $(0.0184)$ & $(0.0178)$ \\
\hline & $(0.50,0.50,0.50,1.00,1.00,1.00)$ & 0.9354 & 0.9822 & 0.9008 & 0.9218 \\
\hline & & $(0.1598)$ & $(0.2103)$ & $(0.1611)$ & $(0.1571)$ \\
\hline \multirow[t]{4}{*}{$(30,30,50,100,100,200)$} & $(0.05,0.05,0.10,0.10,0.15,0.15)$ & 0.9398 & 0.9800 & 0.8678 & 0.9312 \\
\hline & & $(0.0226)$ & $(0.0283)$ & $(0.0236)$ & $(0.0223)$ \\
\hline & $(0.50,0.50,0.50,1.00,1.00,1.00)$ & 0.9284 & 0.9788 & 0.8618 & 0.9100 \\
\hline & & $(0.2257)$ & $(0.2980)$ & $(0.2291)$ & $(0.2216)$ \\
\hline \multirow[t]{4}{*}{$(100,100,100,100,100,100)$} & $(0.05,0.05,0.10,0.10,0.15,0.15)$ & 0.9480 & 0.9830 & 0.9282 & 0.9406 \\
\hline & & $(0.0123)$ & $(0.0158)$ & $(0.0124)$ & $(0.0122)$ \\
\hline & $(0.50,0.50,0.50,1.00,1.00,1.00)$ & 0.9362 & 0.9952 & 0.9166 & 0.9256 \\
\hline & & $(0.1149)$ & $(0.1747)$ & $(0.1154)$ & $(0.1131)$ \\
\hline \multirow[t]{4}{*}{$(200,200,200,200,200,200)$} & $(0.05,0.05,0.10,0.10,0.15,0.15)$ & 0.9512 & 0.9872 & 0.9410 & 0.9428 \\
\hline & & $(0.0087)$ & $(0.0111)$ & $(0.0087)$ & $(0.0086)$ \\
\hline & $(0.50,0.50,0.50,1.00,1.00,1.00)$ & 0.9418 & 0.9968 & 0.9322 & 0.9364 \\
\hline & & $(0.0813)$ & $(0.1231)$ & $(0.0816)$ & $(0.0801)$ \\
\hline \multirow[t]{4}{*}{$(500,500,500,500,500,500)$} & $(0.05,0.05,0.10,0.10,0.15,0.15)$ & 0.9510 & 0.9876 & 0.9440 & 0.9464 \\
\hline & & $(0.0055)$ & $(0.0070)$ & $(0.0055)$ & $(0.0054)$ \\
\hline & $(0.50,0.50,0.50,1.00,1.00,1.00)$ & 0.9520 & 0.9960 & 0.9494 & 0.9494 \\
\hline & & $(0.0514)$ & $(0.0776)$ & $(0.0514)$ & $(0.0506)$ \\
\hline \multirow[t]{4}{*}{$(1000,1000,1000,1000,1000,1000)$} & $(0.05,0.05,0.10,0.10,0.15,0.15)$ & 0.9520 & 0.9890 & 0.9496 & 0.9490 \\
\hline & & $(0.0039)$ & $(0.0049)$ & $(0.0039)$ & $(0.0038)$ \\
\hline & $(0.50,0.50,0.50,1.00,1.00,1.00)$ & 0.9520 & 0.9974 & 0.9488 & 0.9470 \\
\hline & & $(0.0363)$ & $(0.0548)$ & $(0.0363)$ & $(0.0358)$ \\
\hline
\end{tabular}

Table 3. Sample statistics of Northern, Northeastern, Central, Eastern, and Southern regions (mm)

\begin{tabular}{cccccc}
\hline Statistics & Northern region & Northeastern region & Central region & Eastern region & Southern region \\
\hline$n$ & 30 & 31 & 21 & 16 & 27 \\
$\bar{y}$ & 17.1200 & 16.8677 & 17.9333 & 19.9688 & 18.3852 \\
$s_{Y}$ & 13.5250 & 30.9424 & 14.7627 & 15.8678 & 23.2974 \\
$\bar{x}$ & 2.5082 & 1.6238 & 2.2851 & 2.5292 & 1.8608 \\
$s_{X}$ & 0.8945 & 1.6007 & 1.5163 & 1.2827 & 1.7549 \\
$\hat{\theta}$ & 1.1072 & 3.4592 & 2.9942 & 2.0450 & 4.5550 \\
\hline
\end{tabular}

